\title{
On the average number of direct factors of finite abelian groups
}

\author{
by \\ Wenguang Zhai (Jinan) and Xiaodong CaO (Beijing)
}

1. Introduction. Let $t(G)$ denote the number of direct factors of a finite abelian group $G$. We shall be concerned with obtaining estimates for the sum

$$
T(x)=\sum t(G)
$$

where the summation is taken over all abelian groups of order not exceeding $x$. The asymptotic behaviour of $T(x)$ was first studied by E. Cohen [2], who derived

$$
T(x)=d_{1} x(\log x+2 \gamma-1)+d_{2} x+\Delta_{0}(x),
$$

where $\gamma$ is the Euler constant and $\Delta_{0}(x)$ is estimated by

$$
\Delta_{0}(x) \ll \sqrt{x} \log x .
$$

E. Krätzel [5] improved this result to

$$
\Delta_{0}(x)=d_{3} \sqrt{x}\left(\frac{1}{2} \log x+2 \gamma-1\right)+d_{4} \sqrt{x}+\Delta_{1}(x)
$$

with the new remainder term $\Delta_{1}(x)$ satisfying

$$
\Delta_{1}(x) \ll x^{5 / 12} \log ^{4} x .
$$

We remark that in the formulas (1.2) and (1.3) $d_{1}, d_{2}, d_{3}, d_{4}$ are effective constants which will be defined by $(1.5)-(1.8)$ below.

The exponent $5 / 12$ was improved to $83 / 201,45 / 109,3 / 8$ respectively by Menzer [9], Menzer and Seibold [10] and Yu Gang [13]. The latest result is due to Liu [7], who proved that

$$
\Delta_{1}(x) \ll x^{7 / 19+\varepsilon} .
$$

The aim of this paper is further to improve this result. We have

1991 Mathematics Subject Classification: Primary 11N37. 
Theorem 1. Let $d_{1}, d_{2}, d_{3}, d_{4}$ be defined by

$$
\begin{aligned}
& d_{1}=\zeta^{2}(2) \sum_{n=1}^{\infty} \tau_{3}(n) n^{-1}, \\
& d_{2}=-\sum_{n=1}^{\infty} \tau_{3}(n) n^{-1}\left(\zeta^{2}(2) \log n-4 \zeta(2) \zeta^{\prime}(2)\right), \\
& d_{3}=\zeta^{2}\left(\frac{1}{2}\right) \sum_{n=1}^{\infty} \tau_{3}(n) n^{-1 / 2}, \\
& d_{4}=-\sum_{n=1}^{\infty} \tau_{3}(n) n^{-1 / 2}\left(\frac{1}{2} \zeta^{2}\left(\frac{1}{2}\right) \log n-\zeta\left(\frac{1}{2}\right) \zeta^{\prime}\left(\frac{1}{2}\right)\right),
\end{aligned}
$$

where $\tau_{3}(n)$ is defined by

$$
\sum_{n=1}^{\infty} \tau_{3}(n) n^{-s}=\prod_{u=3}^{\infty} \zeta^{2}(u s) \quad\left(\Re s>\frac{1}{3}\right) .
$$

Then we have

$$
\begin{aligned}
T(x)= & d_{1} x(\log x+2 \gamma-1)+d_{2} x+d_{3} \sqrt{x}\left(\frac{1}{2} \log x+2 \gamma-1\right) \\
& +d_{4} \sqrt{x}+O\left(x^{4 / 11+\varepsilon}\right) .
\end{aligned}
$$

Following Krätzel [5], we only need to study the asymptotic behaviour of the divisor function $d(1,1,2,2 ; n)$ which is defined by

$$
d(1,1,2,2 ; n)=\sum_{n=n_{1} n_{2} n_{3}^{2} n_{4}^{2}} 1 .
$$

Let $\Delta(1,1,2,2 ; x)$ denote the error term of the summation function

$$
D(1,1,2,2 ; x)=\sum_{n \leq x} d(1,1,2,2 ; n) .
$$

We then have

THEOREM 2. We have

$$
\Delta(1,1,2,2 ; x)=O\left(x^{4 / 11+\varepsilon}\right) .
$$

Theorem 1 immediately follows from Theorem 2 .

Notations. $e(t)=\exp (2 \pi i t)$. [t] is the integer part of $t$, and $\{t\}=t-[t]$, $\|t\|=\min (\{t\}, 1-\{t\}), n \sim N$ means $N<n \leq 2 N, n \cong N$ means $C_{1} N \leq n \leq C_{2} N$ for some constants $C_{1}$ and $C_{2}$. $\varepsilon$ is a sufficiently small number which may be different at each occurrence. $\Delta(t)$ always denotes the error term of the Dirichlet divisor problem. 
2. A non-symmetric expression of $\Delta(1,1,2,2 ; x)$. In this paper we do not use the symmetric expression of $\Delta(1,1,2,2 ; x)$ due to Vogts [12] (also [10], Lemma 2). We shall use a non-symmetric expression of $\Delta(1,1,2,2 ; x)$ which is easier and simpler. We have the following basic lemma.

BASIC LEMma. We have

$$
\begin{aligned}
\Delta(1,1,2,2 ; x)= & \sum_{m \leq x^{1 / 3}} d(m) \Delta\left(\frac{x}{m^{2}}\right) \\
& +\sum_{m \leq x^{1 / 3}} d(m) \Delta\left(\sqrt{\frac{x}{m}}\right)+O\left(x^{1 / 3} \log x\right) .
\end{aligned}
$$

Proof. We only sketch the proof since it is elementary and direct.

We begin with

$$
\begin{aligned}
D(1,1,2,2 ; x) & =\sum_{n \leq x} d(1,1,2,2 ; n)=\sum_{n_{1} n_{2} n_{3}^{2} n_{4}^{2} \leq x} 1=\sum_{n m^{2} \leq x} d(n) d(m) \\
& =\sum_{n \leq x^{1 / 3}} d(n) D\left(\sqrt{\frac{x}{n}}\right)+\sum_{m \leq x^{1 / 3}} d(m) D\left(\frac{x}{m^{2}}\right)-D^{2}\left(x^{1 / 3}\right) \\
& =\sum_{1}+\sum_{2}-\sum_{3},
\end{aligned}
$$

where $D(u)=\sum_{n \leq u} d(n)$.

Now we use the well-known abelian partial summation formula

$$
\sum_{n \leq u} d(n) f(n)=D(u) f(u)-\int_{1}^{u} D(t) f^{\prime}(t) d t
$$

to $\sum_{1}$ and $\sum_{2}$, and utilize the well-known formula

$$
D(x)=x \log x+(2 \gamma-1) x+\Delta(x)
$$

with $\Delta(x) \ll x^{1 / 3}$. We get

$$
\begin{aligned}
D(1,1,2,2 ; x)= & \text { main terms }+\sum_{n \leq x^{1 / 3}} d(n) \Delta\left(\sqrt{\frac{x}{n}}\right) \\
& +\sum_{m \leq x^{1 / 3}} d(m) \Delta\left(\frac{x}{m^{2}}\right)+O\left(x^{1 / 3} \log x\right),
\end{aligned}
$$

whence our lemma follows. 
3. Some preliminary lemmas. In this section we quote some lemmas to be used later.

Lemma 1. Suppose $0<c_{1} \lambda_{1} \leq\left|f^{\prime}(n)\right| \leq c_{2} \lambda_{1}$ and $\left|f^{\prime \prime}(n)\right| \cong \lambda_{1} N^{-1}$ for $N \leq n \leq C N$. Then

$$
\sum_{n \cong N} e(f(n)) \ll \lambda_{1}^{1 / 2} N^{1 / 2}+\lambda_{1}^{-1}
$$

If $c_{2} \lambda_{1} \leq 1 / 2$, then

$$
\sum_{n \cong N} e(f(n)) \ll \lambda_{1}^{-1} .
$$

Lemma 2. Let $\alpha, \beta$ be real numbers, $\alpha \beta(\alpha+\beta-1)(\alpha+\beta-2) \neq 0$. Let $f(x, y)=A x^{\alpha} y^{\beta}, D \subset\{(x, y) \mid x \sim X, y \sim Y\}, X \geq Y, F \equiv A X^{\alpha} Y^{\beta}$, $N \equiv X Y$. Then

$$
\begin{aligned}
S & \equiv(N F)^{-\varepsilon} \sum_{(x, y) \in D} e(f(x, y)) \\
& \ll \sqrt[6]{F^{2} N^{3}}+N^{5 / 6}+\sqrt[8]{F^{-1} N^{8} X^{-1}}+N F^{-1 / 4}+N Y^{-1 / 2} .
\end{aligned}
$$

Lemma 3. Let $f(x)$ and $g(x)$ be algebraic functions for $x \in[a, b]$, satisfying

$$
\begin{gathered}
\left|f^{\prime \prime}(x)\right| \cong R^{-1}, \quad f^{\prime \prime \prime}(x) \ll(R U)^{-1} \\
|g(x)| \leq H, \quad g^{\prime}(x) \ll H U_{1}^{-1}, \quad U, U_{1} \geq 1 .
\end{gathered}
$$

Then

$$
\begin{aligned}
\sum_{a<n \leq b} g(n) e(f(n))= & \sum_{\alpha<u \leq \beta} b_{u} \frac{g(n(u))}{\sqrt{f^{\prime \prime}(n(u))}} e(f(n(u))-u n(u)+1 / 8) \\
& +O\left(H \log (\beta-\alpha+2)+H(b-a+R)\left(U^{-1}+U_{1}^{-1}\right)\right) \\
& +O\left(H \min \left(R^{1 / 2}, \max \left(\frac{1}{\langle\alpha\rangle}, \frac{1}{\langle\beta\rangle}\right)\right)\right),
\end{aligned}
$$

where $[\alpha, \beta]$ is the image of $[a, b]$ under the mapping $y=f^{\prime}(x), n(u)$ is determined by the equation $f^{\prime}(n(u))=u$, and

$$
b_{u}= \begin{cases}1 & \text { for } \alpha<u<\beta, \\ \frac{1}{2} & \text { for } u=\alpha=\text { integer or } u=\beta=\text { integer } ;\end{cases}
$$

the function $\langle x\rangle$ is defined as follows:

$$
\begin{gathered}
\langle x\rangle= \begin{cases}\|x\| & \text { if } x \text { is not an integer }, \\
\beta-\alpha & \text { otherwise, }\end{cases} \\
\sqrt{f^{\prime \prime}}= \begin{cases}\sqrt{f^{\prime \prime}} & \text { if } f^{\prime \prime}>0, \\
i \sqrt{\left|f^{\prime \prime}\right|} & \text { if } f^{\prime \prime}<0 .\end{cases}
\end{gathered}
$$


Lemma 4. Suppose $f(n) \ll P$ and $f^{\prime}(n) \gg \Delta$ for $n \cong N$. Then

$$
\sum_{n \cong N} \min \left(D, \frac{1}{\|f(n)\|}\right) \ll(P+1)\left(D+\Delta^{-1}\right) \log \left(2+\Delta^{-1}\right) .
$$

Lemma 5. Suppose $a(n)=O(1), 0<L \leq M<N \leq c L, L \gg 1, T \geq 2$. Then

$$
\begin{aligned}
\sum_{M<n \leq N} a(n)= & \frac{1}{2 \pi i} \int_{-T}^{T} \sum_{L<l \leq c L} \frac{a(l)}{l^{i t}} \cdot \frac{N^{i t}-M^{i t}}{t} d t \\
& +O\left(\min \left(1, \frac{L}{T\|M\|}\right)+\min \left(1, \frac{L}{T\|N\|}\right)\right)+O\left(\frac{L \log (1+L)}{T}\right) .
\end{aligned}
$$

Lemma 6. Let $\mathcal{X}$ and $\mathcal{Y}$ be two finite sets of real numbers, $\mathcal{X} \subset[-X, X]$, $\mathcal{Y} \subset[-Y, Y]$. Then for any complex functions $u(x)$ and $v(y)$, we have

$$
\begin{aligned}
& \left|\sum_{x \in \mathcal{X}} \sum_{y \in \mathcal{Y}} u(x) v(y) e(x y)\right|^{2} \\
& \quad \leq 2 \pi^{2}(1+X Y) \sum_{\substack{x \in \mathcal{X}, x^{\prime} \in \mathcal{X} \\
2 Y\left|x-x^{\prime}\right| \leq 1}}\left|u(x) u\left(x^{\prime}\right)\right| \sum_{\substack{y \in \mathcal{Y}, y^{\prime} \in \mathcal{Y} \\
2 X\left|y-y^{\prime}\right| \leq 1}}\left|v(y) v\left(y^{\prime}\right)\right| .
\end{aligned}
$$

Lemma 7. Let $\alpha \beta \neq 0, m \geq 1$ and $N \geq 1$. Let $\mathcal{A}(M, N ; \Delta)$ be the number of quadruples $(m, \widetilde{m}, n, \widetilde{n})$ such that

$$
\left|\left(\frac{\widetilde{m}}{m}\right)^{\alpha}-\left(\frac{\widetilde{n}}{n}\right)^{\beta}\right|<\Delta
$$

with $M \leq m, \widetilde{m} \leq 2 M$ and $N \leq n, \widetilde{n} \leq 2 N$. Then

$$
\mathcal{A}(M, N ; \Delta) \ll M N \log 2 M N+\Delta M^{2} N^{2} .
$$

LEMMA 8. We have

$$
\psi(t)=\sum_{1 \leq|j| \leq J} a(j) e(j t)+O\left(\sum_{|j| \leq J} b(j) e(j t)\right)
$$

with $a_{j} \ll|j|^{-1}$ and $b_{j} \ll J^{-1}$.

Lemmas 1, 5, 4 are Lemmas 1, 2, 3 of C.-H. Jia [4] respectively. Lemmas 6 and 7 are Proposition 1 and Lemma 1 of [3]. Lemma 2 is Lemma 9 of H.-Q. Liu [6]. Lemma 3 is Lemma 1 of [8]. For Lemma 8 see Vaaler [11].

4. Proof of Theorem 2. In order to prove Theorem 2, we only need to estimate the two sums in the Basic Lemma of Section 2.

We first estimate the sum $\sum_{1}=\sum_{m \leq x^{1 / 3}} d(m) \Delta\left(x / m^{2}\right)$. We have

Proposition 1. $\sum_{1}=O\left(x^{4 / 11+\varepsilon}\right)$. 
Proof. We only need to show that

$$
S(M)=\sum_{m \sim M} d(m) \Delta\left(\frac{x}{m^{2}}\right) \ll x^{4 / 11+\varepsilon}
$$

for any $1 \ll M \ll x^{1 / 3}$.

Case $1 . M \ll x^{3 / 11}$. Let

$$
S(M, N)=\sum_{m \sim M} A_{m} \sum_{n \sim N} b_{n} e\left(\frac{2 \sqrt{n x}}{m}\right)
$$

with $A_{m}=d(m) m^{-1 / 2}$ and $b_{n}=d(n) n^{-3 / 4}$.

By the well-known Voronoï formula for $\Delta(u)$ we have

$$
\begin{aligned}
S(M) & \ll x^{1 / 4}\left|\sum_{m \sim M} A_{m} \sum_{n \leq x^{3 / 11}} B_{n} e\left(\frac{2 \sqrt{n x}}{m}\right)\right|+O\left(x^{4 / 11+\varepsilon}\right) \\
& \ll x^{1 / 4} \log x|S(M, N)|+x^{4 / 11+\varepsilon}
\end{aligned}
$$

for some $N \leq x^{3 / 11}$ by a splitting-up argument.

R. C. Baker [1] have estimated the sum $S(M, N)$ with $A_{m}$ replaced by $\mu(m) m^{-1 / 2}$. Applying the same arguments of Baker we can obtain

$$
S(M, N) \ll x^{5 / 44+\varepsilon}
$$

if $N \gg \max \left(1, M^{2} x^{-5 / 11}\right)$.

Now we suppose $M \gg x^{5 / 22}$ and $N \ll M^{2} x^{-5 / 11}$. It suffices for us to bound

$$
T(M, N)=\sum_{m \sim M} a_{m} \sum_{n \sim N} b_{n} e\left(\frac{2 \sqrt{n x}}{m}\right)
$$

with $a_{m}=d(m) M^{1 / 2} m^{-1 / 2}$ and $b_{n}=d(n) N^{3 / 4-\varepsilon} N^{-3 / 4}$. We have

$$
\begin{aligned}
T(M, N) & \ll N\left|\sum_{\substack{M<u v \leq 2 M \\
u \geq v}} e\left(\frac{2 \sqrt{n x}}{u v}\right)\right| \\
& \ll N \sum_{V=2^{j}<(2 M)^{1 / 2}}\left|\sum_{\substack{M<u v \leq 2 M \\
u \geq v \\
V<v \leq 2 V}} e\left(\frac{2 \sqrt{n x}}{u v}\right)\right|,
\end{aligned}
$$

where the sum

$$
\left|\sum_{\substack{M<u v \leq 2 M \\ u \geq v}} e\left(\frac{2 \sqrt{n x}}{u v}\right)\right|
$$


takes the maximal value at $n$. Let $\phi(V)$ denote the inner sum of (4.3). By Lemma 2 we get

(4.4) $\quad x^{-\varepsilon} \phi(V)$

$\ll N^{1 / 6} x^{1 / 6} M^{1 / 6}+M^{5 / 6}+(N x)^{-1 / 16} M^{17 / 16}+(N x)^{-1 / 8} M^{5 / 4}+M V^{-1 / 2}$.

Now we use Lemma 1 to estimate the sum over $u$ and the sum over $v$ trivially. We can obtain

$$
\phi(V) \ll \frac{M^{2}}{\sqrt{N x}}+\frac{N^{1 / 4} x^{1 / 4} V}{M^{1 / 2}} .
$$

From (4.4) and (4.5) we have

$$
\begin{aligned}
x^{-\varepsilon} \phi(V) & \\
\ll & N^{1 / 6} x^{1 / 6} M^{1 / 6}+M^{5 / 6}+(N x)^{-1 / 16} M^{17 / 16}+(N x)^{-1 / 8} M^{5 / 4} \\
& +\frac{M^{2}}{\sqrt{N x}}+\min \left(M V^{-1 / 2}, x^{1 / 4} N^{1 / 4} M^{-1 / 2} V\right) \\
\ll & N^{1 / 6} x^{1 / 6} M^{1 / 6}+M^{5 / 6}+(N x)^{-1 / 16} M^{17 / 16}+(N x)^{-1 / 8} M^{5 / 4} \\
& +\frac{M^{2}}{\sqrt{N x}}+x^{1 / 12} N^{1 / 12} M^{1 / 2} .
\end{aligned}
$$

From (4.3), (4.6) and the definition of $S(M, N)$ we get

$$
\begin{aligned}
x^{-\varepsilon} S(M, N) \ll & x^{1 / 6} M^{-1 / 3} N^{5 / 12}+M^{1 / 3} N^{1 / 4} \\
& +x^{-1 / 16} M^{9 / 16} N^{3 / 16}+x^{-1 / 8} M^{3 / 4} N^{1 / 8}+x^{1 / 12} \\
\ll & x^{5 / 44},
\end{aligned}
$$

where the facts $N \ll M^{2} x^{-5 / 11}$ and $M \ll x^{3 / 11}$ are used.

Thus in any case we always have

$$
S(M, N) \ll x^{5 / 44+\varepsilon},
$$

whence (4.1) follows for the case $M \ll x^{3 / 11}$.

Case 2. $x^{3 / 11} \ll M \ll x^{1 / 3}$. By the formula

$$
\Delta(u)=-2 \sum_{n \leq u^{1 / 2}} \psi(u / n)+O(1)
$$

we have

$$
\begin{aligned}
& S(M)=-2 \sum_{M<m \leq 2 M} d(m) \sum_{m n \leq x^{1 / 2}} \psi\left(\frac{x}{m^{2} n}\right)+O\left(x^{3 / 11}\right)
\end{aligned}
$$

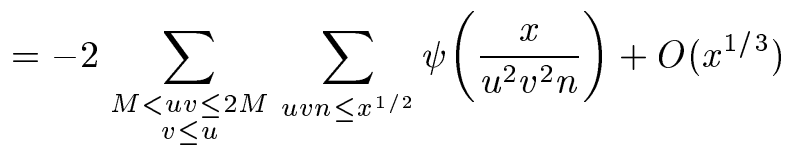




$$
\begin{aligned}
& =-2\left(\sum_{v \leq u \leq n}+\sum_{\substack{v \leq u \\
n<u}}\right)+O\left(x^{1 / 3}\right) \\
& =-2\left(\sum_{v \leq u \leq n}+\sum_{v \leq n<u}+\sum_{n<v \leq u}\right)+O\left(x^{1 / 3}\right) \\
& =-2\left(\sum_{1}+\sum_{2}+\sum_{3}\right)+O\left(x^{1 / 3} \log x\right),
\end{aligned}
$$

say, where we used the fact that if $u=v$ and $n<u$, then $u n<x^{1 / 3}$.

We shall only estimate $\sum_{1} ; \sum_{2}$ and $\sum_{3}$ can be estimated in the same way.

$\sum_{1}$ can be divided into $O\left(\log ^{2} x\right)$ sums of the form

$$
\sum_{1}(V, N)=\sum_{(v, u, n) \in D} \psi\left(\frac{x}{u^{2} v^{2} n}\right)
$$

where

$$
\begin{gathered}
D=\left\{(u, v, n) \mid M<u v \leq 2 M, \text { uvn } \leq x^{1 / 2}, v \leq u \leq n,\right. \\
V<v \leq 2 V, N<n \leq 2 N\} .
\end{gathered}
$$

Let $U=M / V$. By Lemma 8 we get

$$
\begin{aligned}
\sum_{1}(V, N) & \ll \frac{V U N}{J}+\sum_{h \leq J} h^{-1}\left|\sum_{(v, u, n) \in D} e\left(\frac{h x}{u^{2} v^{2} n}\right)\right| \\
& \ll \frac{V U N}{J}+\sum_{H=2^{j}} H^{-1} \sum_{h \sim H}\left|\sum_{(v, u, n) \in D} e\left(\frac{h x}{u^{2} v^{2} n}\right)\right| .
\end{aligned}
$$

Thus it suffices to bound

$$
\phi_{1}(H, V, U, N)=\sum_{h \sim H}\left|\sum_{(v, u, n) \in D} e\left(\frac{h x}{u^{2} v^{2} n}\right)\right| .
$$

Now put

$$
a=\max (N, u), \quad b=\min \left(2 N, \frac{x^{1 / 2}}{u v}\right), \quad \beta=\frac{h x}{u^{2} v^{2} a^{2}}, \quad \alpha=\frac{h x}{u^{2} v^{2} b^{2}} .
$$

Then Lemma 3 yields

$$
\begin{aligned}
\sum_{a \leq n \leq b} e\left(\frac{-h x}{u^{2} v^{2} n}\right)= & c_{0} \sum_{\alpha<r \leq \beta} b_{r} \frac{h^{1 / 4} x^{1 / 4}}{u^{1 / 2} v^{1 / 2} r^{3 / 4}} e\left(\frac{-2 \sqrt{r h x}}{u v}\right) \\
& +O\left(\log x+\min \left(\frac{N}{H^{1 / 2} F^{1 / 2}}, \frac{1}{\langle\alpha\rangle}\right)\right. \\
& \left.+\min \left(\frac{N}{H^{1 / 2} F^{1 / 2}}, \frac{1}{\langle\beta\rangle}\right)\right),
\end{aligned}
$$

where $F=x /\left(V^{2} U^{2} N\right)$. 
We first consider the contribution of the error term of (4.12) to $\phi_{1}(H, V, U, N)$. Obviously, the contribution of $\log x$ is

$$
H V U \log x \ll H x^{1 / 3} \log x .
$$

If $b=x^{1 / 2} /\left(u^{2} v^{2}\right)$, then $\alpha$ is an integer. By Lemma $1,1 /\langle\alpha\rangle \ll 1$, hence the contribution of $\min \left(N /\left(H^{1 / 2} F^{1 / 2}\right), 1 /\langle\alpha\rangle\right)$ to $\phi_{1}(H, V, U, N)$ is $O\left(H x^{1 / 3}\right)$. If $b=2 N$, then $\alpha=h x /\left(4 u^{2} v^{2} N^{2}\right)$, by Lemma 3 (we sum over $u$ ), the contribution of $\min \left(N /\left(H^{1 / 2} F^{1 / 2}\right), 1 /\langle\alpha\rangle\right)$ is

$$
H V \sum_{u \sim U} \min \left(\frac{N}{H^{1 / 2} F^{1 / 2}}, \frac{1}{\left\|\frac{h x}{4 u^{2} v^{2} N^{2}}\right\|}\right) \ll H^{3 / 2} V F^{1 / 2} \log x .
$$

Similarly, the contribution of $\min \left(N /\left(H^{1 / 2} F^{1 / 2}\right), 1 /\langle\beta\rangle\right)$ is

$$
H x^{1 / 3} \log x+H^{3 / 2} V F^{1 / 2} \log x .
$$

From (4.11)-(4.15) we have

$$
\begin{aligned}
\phi_{1}(H, V, U, N)= & \sum_{h \sim H} c(h) \sum_{(v, u, n) \in D} e\left(\frac{h x}{u^{2} v^{2} n}\right) \\
= & c_{0} \sum_{h \sim H} c(h) \sum_{(v, u)} \sum_{\alpha<r \leq \beta} \frac{b_{r} h^{1 / 4} x^{1 / 4}}{u^{1 / 2} v^{1 / 2} r^{3 / 4}} e\left(\frac{-2 \sqrt{r h x}}{u v}\right) \\
& +O\left(H x^{1 / 3} \log x+H^{3 / 2} V F^{1 / 2} \log x\right),
\end{aligned}
$$

where $|c(h)| \leq 1$.

Now we first use Lemma 5 to the variable $r$ and then to the variable $u$ (or we can use the same argument of (13) of Liu [8]). We get

$$
\begin{aligned}
\phi_{1}(H, V, U, N) \ll & \frac{N}{H^{1 / 2} F^{1 / 2}} \sum_{h \sim H} \sum_{u \sim U}\left|\sum_{(v, r) \in D_{2}} C(v, r) e\left(\frac{-2 \sqrt{r h x}}{u v}\right)\right| \\
& +O\left(H x^{1 / 3} \log x+H^{3 / 2} V F^{1 / 2} \log x\right),
\end{aligned}
$$

where we used the fact that the contribution of the error term when we used Lemma 5 is $O\left(H x^{1 / 3} \log x+H^{3 / 2} V F^{1 / 2} \log x\right)$ and $D_{2}=\{(v, r) \mid v \sim V$, $\left.r \cong H F N^{-1}=R\right\}$.

By Lemma 6 we get

$$
\sum_{h \sim H} \sum_{u \sim U}\left|\sum_{(v, r) \in D_{2}} C(v, r) e\left(\frac{-2 \sqrt{r h x}}{u v}\right)\right| \ll\left(H F B_{1} B_{2}\right)^{1 / 2}
$$

where $B_{1}$ is the number of lattice points $(h, u, \widetilde{h}, \widetilde{u})$ such that

$$
h, \widetilde{h} \sim H, \quad u, \widetilde{u} \cong U, \quad\left|\frac{\sqrt{h}}{u}-\frac{\sqrt{\widetilde{h}}}{\widetilde{u}}\right| \ll \frac{V}{\sqrt{R x}},
$$


and where $B_{2}$ is the number of lattice points $(v, r, \widetilde{v}, \widetilde{r})$ such that

$$
v, \widetilde{v} \sim V, \quad r, \widetilde{r} \cong R, \quad\left|\frac{\sqrt{r}}{v}-\frac{\sqrt{\widetilde{r}}}{\widetilde{v}}\right| \ll \frac{U}{\sqrt{H x}} .
$$

By Lemma 7 we have

$$
\begin{aligned}
& B_{1} \ll H U \log x+\frac{1}{H F} U^{2} H^{2} \ll H U \log x, \\
& B_{2} \ll R V \log x+\frac{1}{H F} R^{2} V^{2} \ll R V \log x .
\end{aligned}
$$

Combining (4.17)-(4.20) we get

$$
x^{-\varepsilon} \phi_{1}(H, V, U, N) \ll H(F V U N)^{1 / 2}+x^{1 / 3}+H^{3 / 2} V F^{1 / 2} .
$$

Inserting (4.21) into (4.10) and choosing $J=\left(F^{-1} U^{2} N^{3}\right)^{1 / 3}$, we get

$$
\begin{aligned}
x^{-\varepsilon} \sum_{1}(V, N) & \ll(F V U N)^{1 / 2}+\sqrt[3]{F V^{3} U N}+x^{1 / 3} \\
& \ll(F V U N)^{1 / 2}+x^{1 / 3} \ll(x / M)^{1 / 2}+x^{1 / 3} \ll x^{4 / 11} .
\end{aligned}
$$

In the last step the fact that $M \gg x^{3 / 11}$ was used. From (4.22) we immediately have

In the same way we can show that

$$
\sum_{1} \ll x^{4 / 11+\varepsilon}
$$

$$
x^{-\varepsilon}\left(\sum_{2}+\sum_{3}\right) \ll x^{1 / 2} M^{-1 / 2}+x^{1 / 3} \ll x^{4 / 11} .
$$

Now (4.1) follows from (4.22) and (4.23) in the case $x^{3 / 11} \ll M \ll x^{1 / 3}$. This completes the proof of Proposition 1.

As for $\sum_{2}=\sum_{m \leq x^{1 / 3}} d(m) \Delta(\sqrt{x / m})$, we have the following

Proposition 2. $\sum_{2}=O\left(x^{1 / 3+\varepsilon}\right)$.

Proof. The proof is the same as the proof of Case 2 in Proposition 1, so we omit the details. Actually, similar to the proof of Case 2, we can get (for $x^{1 / 5} \ll M \ll x^{1 / 3}$ )

$$
T(M)=\sum_{m \sim M} d(m) \Delta(\sqrt{x / m}) \ll x^{1 / 4+\varepsilon} M^{1 / 4}+x^{1 / 3} \ll x^{1 / 3+\varepsilon} .
$$

For $M \ll x^{1 / 5}$ we use $\Delta(t) \ll t^{1 / 3}$ and obtain

$$
T(M) \ll \sum_{m \sim M} d(m) x^{1 / 6} M^{-1 / 6} \ll x^{1 / 3} \log x .
$$

Thus Proposition 2 holds.

Now, Theorem 2 follows immediately from the Basic Lemma and the two propositions. 
Acknowledgements. The authors thank the referee for his valuable suggestions.

\section{References}

[1] R. C. Baker, The square-free divisor problem II, Quart. J. Math. Oxford Ser. (2) 47 (1996), 133-146.

[2] E. Cohen, On the average number of direct factors of a finite abelian group, Acta Arith. 6 (1960), 159-173.

[3] E. Fouvry and H. Iwaniec, Exponential sums with monomials, J. Number Theory 33 (1989), 311-333.

[4] C.-H. Jia, On the distribution of square-free numbers (II), Sci. China Ser. A 8 (1992), 812-827.

[5] E. Krätzel, On the average number of direct factors of a finite Abelian group, Acta Arith. 51 (1988), 369-379.

[6] H.-Q. Liu, On the number of abelian groups of a given order, ibid. 59 (1991), 261-277.

[7] -, Divisor problems of 4 and 3 dimensions, ibid. 73 (1995), 249-269.

[8] - The distribution of 4-full numbers, ibid. 67 (1994), 165-176.

[9] H. Menzer, Vierdimensionale Gitterpunktprobleme I, II, Forschungsergebnisse, FSU, Jena, N/89/38, N/89/02.

[10] H. Menzer and R. Seibold, On the average number of direct factors of a finite Abelian group, Monatsh. Math. 110 (1990), 63-72.

[11] J. D. Vaaler, Some extremal problems in Fourier analysis, Bull. Amer. Math. Soc. 12 (1985), 183-216.

[12] M. Vogts, Many-dimensional generalized divisor problems, Math. Nachr. 124 (1985), 103-121.

[13] G. Y u, On the number of direct factors of finite abelian groups, Acta Math. Sinica 37 (1994), 663-670.

Department of Mathematics

Shandong Normal University

Jinan, Shandong 250014

P.R. China

E-mail: arith@sdunetnms.sdu.edu.cn
Beijing Petro-Chemical Engineering College Daxing, Beijing 102600 P.R. China

Received on 15.7.1996

and in revised form on 20.3.1997 